\title{
Open and Hidden Charm Production at RHIC and LHC $^{*}$
}

\author{
R. Vogt \\ Lawrence Berkeley National Laboratory, Berkeley, CA 94720, USA \\ Physics Department, University of California, Davis, CA 95616, USA
}

\begin{abstract}
.
We discuss aspects of open and hidden charm production in hadron-nucleus collisions at RHIC and LHC energies. We first discuss the extraction of the total charm cross section in lower energy collisions and how it compares to next-to-leading order quantum chromodynamics calculations. We then describe calculations of the transverse momentum distributions and their agreement with the shape of the measured STAR transverse momentum distributions. We next explain how shadowing and moderate nuclear absorption can explain the PHENIX $J / \psi \mathrm{dAu} / p p$ ratios.
\end{abstract}

\section{Open charm production at RHIC}

Open charm measurements date back to the late 1970s when $D$ and $\bar{D}$ mesons were first detected, completing the picture of the fourth quark begun when the $J / \psi$ was detected in $p \mathrm{Be}$ and $e^{+} e^{-}$interactions. The charm quark was postulated to have a mass between 1.2 and $1.8 \mathrm{GeV}$, within the regime of perturbative quantum chromodynamics (pQCD). Because of its relatively large mass, it is possible to calculate a total $c \bar{c}$ cross section, not the case for lighter flavors such as strangeness. Charm hadrons are usually detected two ways. The reconstruction of decays to charged hadrons such as $D^{0} \rightarrow K^{-} \pi^{+}(3.8 \%)$ and $D^{+} \rightarrow K^{-} \pi^{+} \pi^{+}(9.1 \%)$ gives the full momentum of the initial $D$ meson, yielding the best direct measurement. Charm can also be detected indirectly via semi-leptonic decays to leptons such as $D \rightarrow K l \nu_{l}$ although the momentum of the parent $D$ meson remains unknown. Early measurements of prompt leptons in beam dump experiments assumed that the density of the dump was high enough to absorb semi-leptonic decays of non-charm hadrons, leaving only the charm component. At modern colliders, it is not possible to use beam dumps to measure charm from leptons but, at sufficiently high $p_{T}$, electrons from charm emerge from hadronic cocktails [1,2].

Although $D$ mesons are usually used to determine the total $c \bar{c}$ cross section, other charm hadrons also exist. The excited $D$ states, $D^{*}$ s, decay primarily to charged and

* This work was supported in part by the Director, Office of Energy Research, Division of Nuclear Physics of the Office of High Energy and Nuclear Physics of the U. S. Department of Energy under Contract Number DE-AC03-76SF00098. 
neutral $D$ mesons. The charm-strange meson, the $D_{s}$, decays to charged hadrons and simultaneously to leptons. The lowest lying charm baryon is the $\Lambda_{c}^{+}$which decays primarily to $\Lambda(u d s)$ but also decays to $p K^{-} \pi^{+}$and to the lepton channel. The heavier ground state charm baryons and their excited states $\left(\Sigma_{c}\right.$ and higher $)$ decay through the $\Lambda_{c}$ channel. The charm-strange baryons are assumed to be a negligible contribution to the total cross section. A selection of charm hadrons, their masses, decay lengths and branching ratios to leptons and charged hadrons are given in Table 1.

\begin{tabular}{ccccc}
$C$ & Mass $(\mathrm{GeV})$ & $c \tau(\mu \mathrm{m})$ & $B(C \rightarrow l X)(\%)$ & $B(C \rightarrow$ Hadrons $)(\%)$ \\
\hline$D^{+}(c \bar{d})$ & 1.869 & 315 & 17.2 & $K^{-} \pi^{+} \pi^{+}(9.1)$ \\
$D^{-}(\bar{c} d)$ & 1.869 & 315 & 17.2 & $K^{+} \pi^{-} \pi^{-}(9.1)$ \\
$D^{0}(c \bar{u})$ & 1.864 & 123.4 & 6.87 & $K^{-} \pi^{+}(3.8)$ \\
$\overline{D^{0}}(\bar{c} u)$ & 1.864 & 123.4 & 6.87 & $K^{+} \pi^{-}(3.8)$ \\
$D^{* \pm}$ & 2.010 & & & $D^{0} \pi^{ \pm}(67.7), D^{ \pm} \pi^{0}(30.7)$ \\
$D^{* 0}$ & 2.007 & & 8 & $D^{+} K^{-} \pi^{+}(4.4), \pi^{+} \pi^{+} \pi^{-}(1.0)$ \\
\hline$D_{s}^{+}(c \bar{s})$ & 1.969 & 147 & 8 & $K^{+} K^{-} \pi^{-}(4.4), \pi^{+} \pi^{-} \pi^{-}(1.0)$ \\
$D_{s}^{-}(\bar{c} s)$ & 1.969 & 147 & 4.5 & $\Lambda X(35), p K^{-} \pi^{+}(2.8)$ \\
\hline$\Lambda_{c}^{+}(u d c)$ & 2.285 & 59.9 & & $\Lambda_{c}^{+} \pi^{+}(100)$ \\
$\Sigma_{c}^{++}(u u c)$ & 2.452 & & & $\Lambda_{c}^{+} \pi^{0}(100)$ \\
$\Sigma_{c}^{+}(u d c)$ & 2.451 & & & $\Lambda_{c}^{+} \pi^{-}(100)$ \\
$\Sigma_{c}^{0}(d d c)$ & 2.452 & & & $\Sigma^{+} K^{-} \pi^{+}(1.18)$ \\
$\Xi_{c}^{+}(u s c)$ & 2.466 & 132 & $\Xi^{-} \pi^{+}(\mathrm{seen})$ \\
$\Xi_{c}^{0}(d s c)$ & 2.472 & 29 & & \\
\hline
\end{tabular}

Table 1. Ground state charm hadrons with their masses, decay lengths (when given) and branching ratios to leptons (when applicable) and some prominent charged hadron decays.

Extracting the total charm cross section is a non-trivial task. To go from a finite number of measured $D$ mesons in a particular decay channel to the total $c \bar{c}$ cross section one must: divide by the branching ratio; correct for the luminosity, $\sigma_{D}=N_{D} / \mathcal{L} t$; extrapolate to full phase space from the finite detector acceptance; divide by two to get the pair cross section from the single $D$ s; and multiply by a correction factor [8] to account for the unmeasured charm hadrons. There are assumptions all along the way. The most important is the extrapolation to full phase space. Before QCD calculations were available, the data were extrapolated assuming a power law for the $x_{F}$ distribution, related to the longitudinal momentum of the charm hadron by $x_{F}=p_{z} /(\sqrt{S} / 2)=2 m_{T} \sinh y / \sqrt{S}$. The canonical parameterization is $\left(1-x_{F}\right)^{c}$ where $c$ was either fit to data over a finite $x_{F}$ range or simply assumed. These parameterizations could lead to large overestimates of the total cross section when $0<c<2$ was assumed, especially when data were taken only near $x_{F}=0$. Lepton measurements were more conservative but were typically at more forward $x_{F}$. 


\subsection{Total cic cross section}

There has been a great deal of improvement over the last 10-15 years. Next-toleading order (NLO) calculations are used in the phase space extrapolation, resulting in considerably less ambiguity in the shape of the $x_{F}$ distributions, $d \sigma / d x_{F}$. The transverse momentum distributions are more difficult, as we will discuss later. To calculate the total cross section to NLO, scaling functions [3] proportional to logs of $\mu^{2} / \mathrm{m}^{2}$ are useful where $\mu$ is the scale of the hard process. The hadronic cross section in $p p$ collisions can be written as

$$
\sigma_{p p}\left(S, m^{2}\right)=\sum_{i, j=q, \bar{q}, g} \int d x_{1} d x_{2} f_{i}^{p}\left(x_{1}, \mu_{F}^{2}\right) f_{j}^{p}\left(x_{2}, \mu_{F}^{2}\right) \widehat{\sigma}_{i j}\left(s, m^{2}, \mu_{F}^{2}, \mu_{R}^{2}\right)
$$

where $x_{1}$ and $x_{2}$ are the fractional momenta carried by the colliding partons and $f_{i}^{p}$ are the proton parton densities. The partonic cross sections are

$$
\begin{aligned}
\widehat{\sigma}_{i j}\left(s, m, \mu_{F}^{2}, \mu_{R}^{2}\right) & =\frac{\alpha_{s}^{2}\left(\mu_{R}^{2}\right)}{m^{2}}\left\{f_{i j}^{(0,0)}(\rho)\right. \\
& \left.+4 \pi \alpha_{s}\left(\mu_{R}^{2}\right)\left[f_{i j}^{(1,0)}(\rho)+f_{i j}^{(1,1)}(\rho) \ln \left(\frac{\mu_{F}^{2}}{m^{2}}\right)\right]+\mathcal{O}\left(\alpha_{s}^{2}\right)\right\}
\end{aligned}
$$

with $s$ the squared partonic center of mass energy, $\rho=4 m^{2} / s$ and $f_{i j}^{(k, l)}$ are the scaling functions given to NLO in Ref. [3]. It is most consistent to assume that the factorization scale, $\mu_{F}$, and the renormalization scale, $\mu_{R}$, are equal, $\mu=\mu_{F}=\mu_{R}$. There is no dependence on the kinematic variables. Some NNLO calculations are available near threshold, $s=x_{1} x_{2} S \sim 1.3\left(4 m^{2}\right)$, applicable only for $\sqrt{S} \leq 20-25 \mathrm{GeV}[4,5]$. The NLO corrections to the leading order (LO) cross sections are relatively large, $K^{(1)}=\sigma_{\mathrm{NLO}} / \sigma_{\mathrm{LO}} \sim 2-3$, depending on $\mu, m$ and the parton densities [6]. The NNLO corrections are about as large to next-to-next-to-leading logarithm [4] but decrease to less than $K^{(1)}$ when subleading logs are included [5]. This $K$ factor is large because, in the range $1.2<m<1.8 \mathrm{GeV}, m<\mu<2 m$ with a 5 -flavor QCD scale, $\Lambda_{5}$, of $0.153 \mathrm{GeV}$ for the GRV98 $\mathrm{HO}$ and $0.22 \mathrm{GeV}$ for the MRST parton densities, $0.21<\alpha_{s}(c)<0.4$, nearly a factor of two variation. (The larger value corresponds to the smallest $m$ and $\mu$ values with the larger $\Lambda_{5}$.) The situation improves for bottom where $\alpha_{s}$ is smaller, $0.16<\alpha_{s}(b)<0.28$, and is quite good for top, $0.092<\alpha_{s}(t)<0.12$. Instead of presenting a wide range of possible cross sections and emphasizing the uncertainties, the approach taken in Ref. [7] has been to "fit" $m$ and $\mu$ for a particular parton density and extrapolate to higher energies. The results are compared to some of the total cross section data [8] on the left-hand side of Fig. 1. The data tend to favor lower values of $m, 1.2-1.3 \mathrm{GeV}$. The two curves cross each other because the MRST calculation with $\mu=2 m$ increases faster at large $\sqrt{S}$ and smaller $x$ due to the stronger QCD evolution of the parton densities at the higher scale. Although the fixed target results are in good agreement with the calculations, the PHENIX point [9] at $130 \mathrm{GeV}$, from $\mathrm{Au}+\mathrm{Au}$ electron measurements, and the STAR point [10], from a combination of electron and reconstructed $D$ measurements, are generally above the calculations. The STAR point is about a factor of four over the calculated cross section. The higher energy $p \bar{p}$ data 
from UA1 [11] and CDF [12] are in better agreement with the calculations. (At these energies, the $p p$ and $p \bar{p}$ cross sections differ by less than $1 \%$ for $\sqrt{S} \geq 630 \mathrm{GeV}$.)
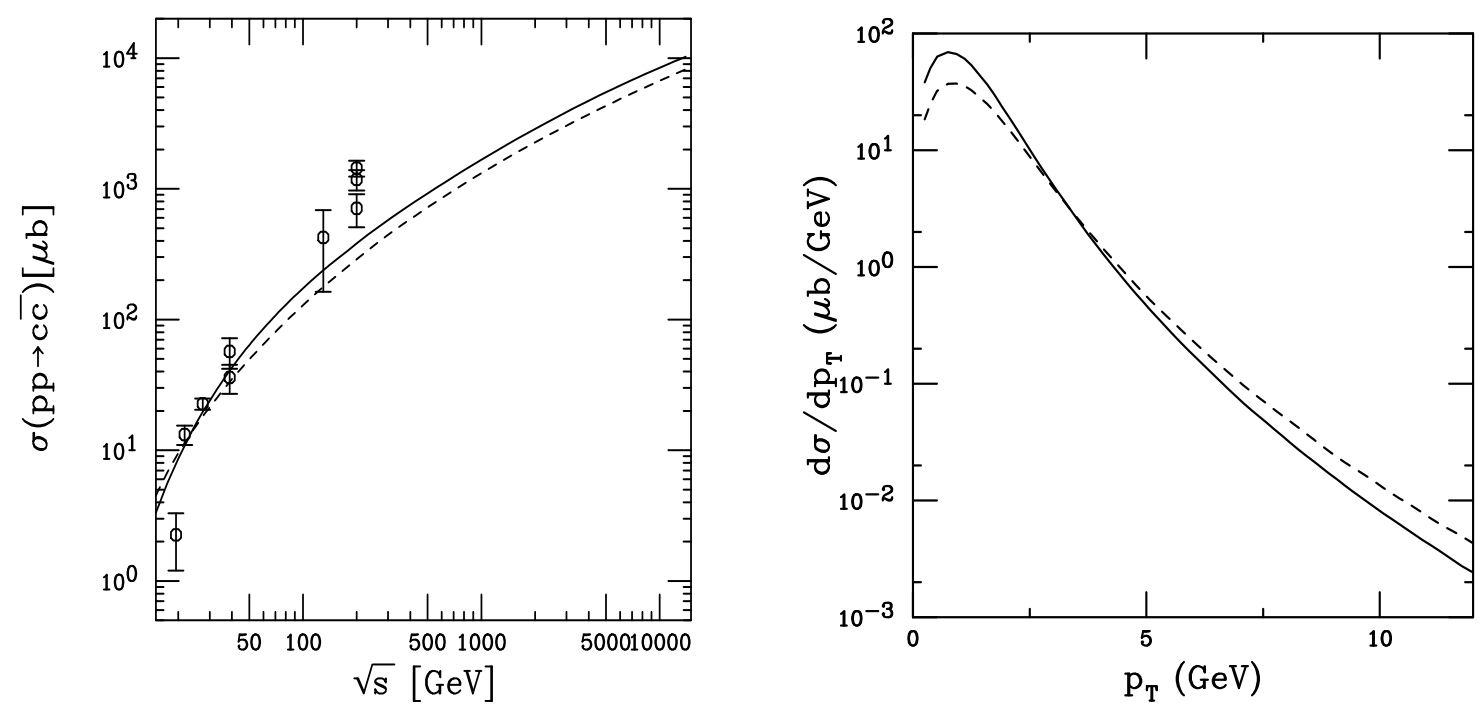

Figure 1. The NLO total $c \bar{c}$ cross sections as a function of $\sqrt{S}$ (left-hand side) and charm quark $p_{T}$ distribution at $\sqrt{S}=200 \mathrm{GeV}$ in the range $|y| \leq 1$ (right-hand side) in $p p$ interactions. The curves are MRST HO (solid) with $m=1.2 \mathrm{GeV}$ and $\mu^{2}=4 m^{2}$ and GRV98 HO (dashed) with $m=1.3 \mathrm{GeV}$ and $\mu^{2}=m^{2}$.

\subsection{Open charm transverse momentum distributions}

Now we turn to the transverse momentum, $p_{T}$, distributions. In this case, the quark mass is no longer the only scale and $p_{T}$-dependent logs also appear. Thus, to interpolate between the high $p_{T}$ scale of $p_{T}$ and the low $p_{T}$ scale of $m$, a scale proportional to $m_{T}$, the transverse mass, is the natural choice. The charm quark $p_{T}$ distributions are not strongly dependent on quark mass for $p_{T} \geq 3 \mathrm{GeV}$, as may be expected, where the difference in rate is $\approx 20 \%$ between $m=1.2$ and $1.8 \mathrm{GeV}$. The difference in the total cross sections is almost all at $p_{T} \leq 3 \mathrm{GeV}$. Changing the scale changes the slope of the $p_{T}$ distributions. The distributions are harder for $\mu=m$ than $\mu=2 m$. The average $p_{T}$, $\left\langle p_{T}\right\rangle$, increases with $m$ and is larger for $\mu=m$.

More modeling is involved for $D$ mesons in the treatment of fragmentation/hadronization and momentum broadening. If factorization holds in the final state (universal fragmentation functions) as well as in the initial state (universal parton distributions) then the fragmentation functions extracted in $e^{+} e^{-}$should also be applicable to $p p$ and $\mathrm{d} A$. However, this assumption does not work well for charm. The Peterson function, generally used in hadroproduction codes, reduces the charm hadron momentum by 30\% relative to the charm quark. As shown in Huang's talk [13], the Peterson function agrees reasonably well with the $e^{+} e^{-}$data. (However, it does not include any scale evolution. In low $\sqrt{S}$ collisions, the momentum reduction due to fragmentation can be compensated by intrinsic transverse momentum, $k_{T}$, broadening. However, such 
broadening cannot compensate the $x_{F}$ distributions, only marginally affected by $k_{T}$ smearing. We have previously shown that the $D$ meson $x_{F}$ distributions are consistent with no momentum loss during charm quark hadronization [14].) The exclusive NLO $Q \bar{Q}$ code of Ref. [15] includes fragmentation and broadening. This program adds the $k_{T}$ kick in the final, rather than the initial state. The initial $k_{T}$ of the partons could have alternatively been given to the entire final-state system, as is essentially done if applied in the initial state, instead of to the $Q \bar{Q}$ pair. The Gaussian function $g_{p}\left(k_{T}\right)$,

$$
g_{p}\left(k_{T}\right)=\frac{1}{\pi\left\langle k_{T}^{2}\right\rangle_{p}} \exp \left(-k_{T}^{2} /\left\langle k_{T}^{2}\right\rangle_{p}\right)
$$

multiplies the parton distribution functions, assuming the $x$ and $k_{T}$ dependencies completely factorize. If true, it does not matter whether the $k_{T}$ dependence appears in the initial or final state. There is no difference if the calculation is LO but at NLO an additional light parton appears in the final state. The difference in the two methods is rather small if $k_{T}^{2} \leq 2 \mathrm{GeV}^{2}$ [8]. The value $\left\langle k_{T}^{2}\right\rangle_{p}=1 \mathrm{GeV}^{2}$ was used in Ref. [8].

The effects of fragmentation and intrinsic $k_{T}$ broadening of $\left\langle k_{T}^{2}\right\rangle=1 \mathrm{GeV}^{2}$ compensate each other at $\sqrt{S}=20 \mathrm{GeV}$ to give a $D$ meson $p_{T}$ distribution very similar to that of the charm quark [13]. However, at RHIC energies, the situation is quite different. Due to the higher $\left\langle p_{T}\right\rangle$ at larger $\sqrt{S}$, the effect of broadening is relatively small and cannot compensate for the momentum loss induced by fragmentation. Interestingly enough, the STAR $D$ and $D^{*} p_{T}$ distribution agrees rather well with the calculated NLO charm quark distribution, as shown in van Leeuwen's talk [16]. On the right-hand side of Fig. 1, we show the corresponding $p_{T}$ distributions at $\sqrt{S}=200 \mathrm{GeV}$ for the two sets of parameters in the total cross section curves on the left-hand side. The differences in the slopes are due to the different scales while the normalization difference is due to the choice of charm mass and the parton densities - the MRST densities generally give a larger cross section due to their larger $\alpha_{s}$. However, the curves need to be scaled up by a factor of four to agree with the STAR normalization [16], as may be expected from the total cross section results. The shape of the charm quark $p_{T}$ distribution at $\sqrt{S}=1.96 \mathrm{TeV}$ also agrees quite well with the CDF data from the Tevatron [12]. Given the large discrepancy between the pQCD result and the STAR cross section, it might be surprising that the normalization is also in good agreement with the sum of the charged and neutral $D$ data scaled to include $D_{s}$ and $\Lambda_{c}$ production. No total cross section is available because only charm hadrons with $p_{T}>5 \mathrm{GeV}$ have been measured so far.

Other model calculations of charm production at collider energies are available. The FONLL calculation [17] resums $\operatorname{logs}$ at $p_{T} \gg m$, resulting in a softer charm quark distribution. Using a harder fragmentation function, fit from $e^{+} e^{-}$data, agreement with the CDF data is obtained. It also underestimates the low $p_{T}$ STAR data since the total cross section is similar to that of NLO pQCD [18]. A calculation with unintegrated gluon distributions and $k_{T}$-dependent matrix elements, assuming saturation behavior at low $x$, has also been made [19]. However, the $x$ values of the STAR data are not really very low. At RHIC, from kinematics $x \sim 0.01$ at $y=1$ and $p_{T}=0$, the highest rapidity measured by STAR by kinematics alone. In reality, the actual $\langle x\rangle$ may be higher when 
weighted by the parton densities. At higher $p_{T}, x$ is larger still, suggesting that the applicability of small $x$ physics for charm at RHIC is rather dubious.

Finally, we would like to discuss reasons why fragmentation does not seem to factorize for charm, as expected. Factorization breaking has been suggested from studies of the $x_{F}$ distributions of e.g. $D^{+}$and $D^{-}$production, particularly in $\pi^{-} A$ interactions where the $D^{-}$is leading relative to the $D^{+}$since the $D^{-}$shares a valence quark with the $\pi^{-}$while the $D^{+}$does not. Several mechanisms such as intrinsic charm [14] and string drag have been proposed, both of which involve charm quark coalescence with spectators. Such comoving partons are naturally produced in a hard scattering. Although it is not intuitive to expect coalescence to work at high $p_{T}$, it seems to do so for charm.

\section{Nuclear dependence of $J / \psi$ production at $\mathbf{R H I C}$ and $\mathbf{L H C}$}

We now turn to $J / \psi$ production in $\mathrm{d}+\mathrm{Au}$ interactions at RHIC. Previously, we calculated the effect of shadowing alone on the $J / \psi \mathrm{d} A / p p$ ratio as a function of rapidity and impact parameter [20]. The large $c \bar{c}$ total cross section also has implications for the $J / \psi$ yield if $J / \psi$ 's arise from $c \bar{c}$ recombination in a QGP. Such a total cross section would suggest significant secondary $J / \psi$ production at RHIC, leading to enhancement rather than suppression in central collisions. There is no evidence for a strong regeneration effect in the PHENIX Au+Au data so far, see Thews' talk [21].

Shadowing, the modification of the parton densities in the nucleus with respect to the free nucleon, is parameterized as $F_{i}^{A}\left(x, \mu^{2}, \vec{b}, z\right)=S^{i}\left(A, x, \mu^{2}, \vec{b}, z\right) f_{i}^{p}\left(x, \mu^{2}\right)$ in Eq. (1). We did not discuss the effect of shadowing on the charm $p_{T}$ distributions because the effect at midrapidity is small and, on the logarithmic scale of the $p_{T}$ distributions, negligible. The $J / \psi$ is another story due to the PHENIX muon capability at forward and backward rapidity. As shown in Pereira's talk [2], although the PHENIX $J / \psi$ data are consistent with shadowing alone, the data are also consistent with nuclear shadowing plus a small absorption cross section of $1-3 \mathrm{mb}$, smaller than that currently obtained in SPS measurements [22]. We have calculated $J / \psi$ production in the color evaporation model (CEM) using the same mass and scale as in $c \bar{c}$ production but cutting off the invariant mass of the pair at $4 m_{D}^{2}$. The calculations of the $\mathrm{d} A / p p$ ratios are done at LO to simplify the calculations. As shown in Fig. 2, the LO and NLO ratios are equivalent. We have now also implemented nucleon absorption in the calculation, showing the effect of several absorption and production mechanisms.

To implement nuclear absorption on $J / \psi$ production in $p A$ collisions, the $p N$ production cross section is weighted by the survival probability, $S^{\text {abs }}$, so that [23]

$$
\sigma_{p A}=\sigma_{p N} \int d^{2} b \int_{-\infty}^{\infty} d z \rho_{A}(b, z) S^{\mathrm{abs}}(b, z)
$$

where $b$ is the impact parameter and $z$ is the longitudinal production point. If $S^{\text {abs }}=1$, $\sigma_{p A}=A \sigma_{p N}$. For $S^{\text {abs }} \neq 1, \sigma_{p A}=A^{\alpha} \sigma_{p N}$. We define $S^{\text {abs }}$ as

$$
S^{\mathrm{abs}}(b, z)=\exp \left\{-\int_{z}^{\infty} d z^{\prime} \rho_{A}\left(b, z^{\prime}\right) \sigma_{\mathrm{abs}}\left(z^{\prime}-z\right)\right\} \text {. }
$$




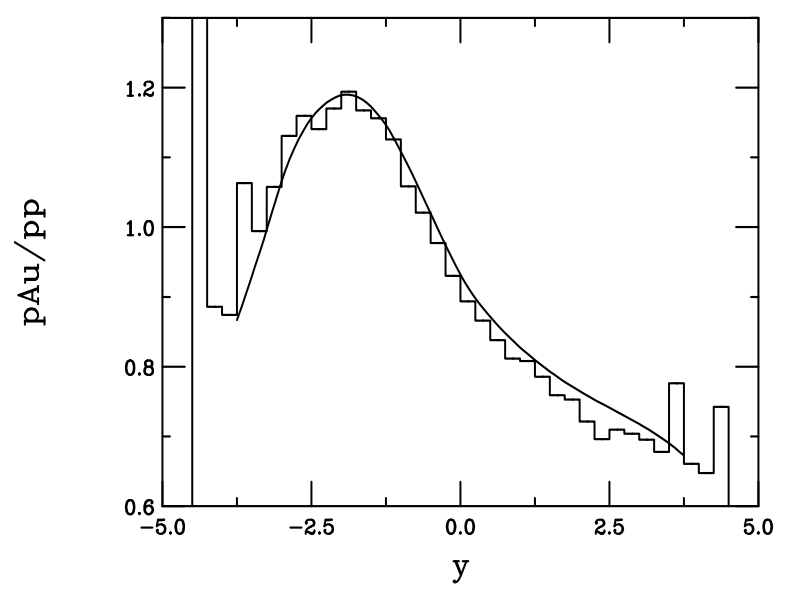

Figure 2. The $J / \psi p \mathrm{Au} / p p$ ratio at $200 \mathrm{GeV}$. We compare the NLO (solid histogram, MRST HO) and LO (solid curve, MRST LO) results using $m=\mu / 2=1.2 \mathrm{GeV}$ with the EKS98 parameterization.

The nucleon absorption cross section, $\sigma_{\mathrm{abs}}$, depends on where the state is produced and how far it travels through nuclear matter. The effective $A$ dependence is obtained from Eqs. (4) and (5) by integrating over $z^{\prime}, z$, and $b$. The contribution to the full $A$ dependence in $\alpha\left(x_{F}\right)$ from absorption alone is only constant if $\sigma_{\text {abs }}$ is constant and independent of the production mechanism [23]. The observed $J / \psi$ yield includes feed down from $\chi_{c J}$ and $\psi^{\prime}$ decays, giving

$$
S_{J / \psi}^{\mathrm{abs}}(b, z)=0.58 S_{J / \psi, \operatorname{dir}}^{\mathrm{abs}}(b, z)+0.3 S_{\chi_{c J}}^{\mathrm{abs}}(b, z)+0.12 S_{\psi^{\prime}}^{\mathrm{abs}}(b, z) .
$$

In color singlet production, the final state absorption cross section depends on the size of the $c \bar{c}$ pair as it traverses the nucleus, allowing absorption to be effective only while the cross section is growing toward its asymptotic size inside the target. On the other hand, if the $c \bar{c}$ is only produced as a color octet, hadronization will occur only after the pair has traversed the target except at very backward rapidity. We have considered a constant octet cross section, as well as one that reverts to a color singlet at backward

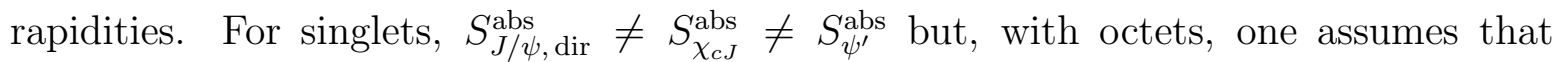

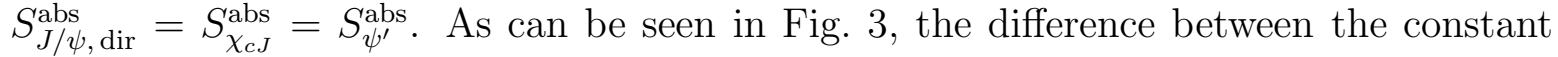
and growing octet assumptions is quite small at large $\sqrt{S}$ with only a small singlet effect at $y<-2$ and -5 at RHIC and the LHC respectively. Singlet absorption is also important only at similar rapidities and is otherwise not different from shadowing alone. Finally, we have also considered a combination of octet and singlet absorption in the context of the NRQCD model, see Ref. [23] for more details. The combination of nonperturbative singlet and octet parameters changes the shape of the shadowing ratio slightly. The results are shown integrated over impact parameter for the EKS98 shadowing parameterization since it gives good agreement with the trend of the PHENIX data.

We will not discuss the spatial dependence of shadowing and absorption in any detail here. The spatial dependence of shadowing alone was discussed in Ref. [20]. When 

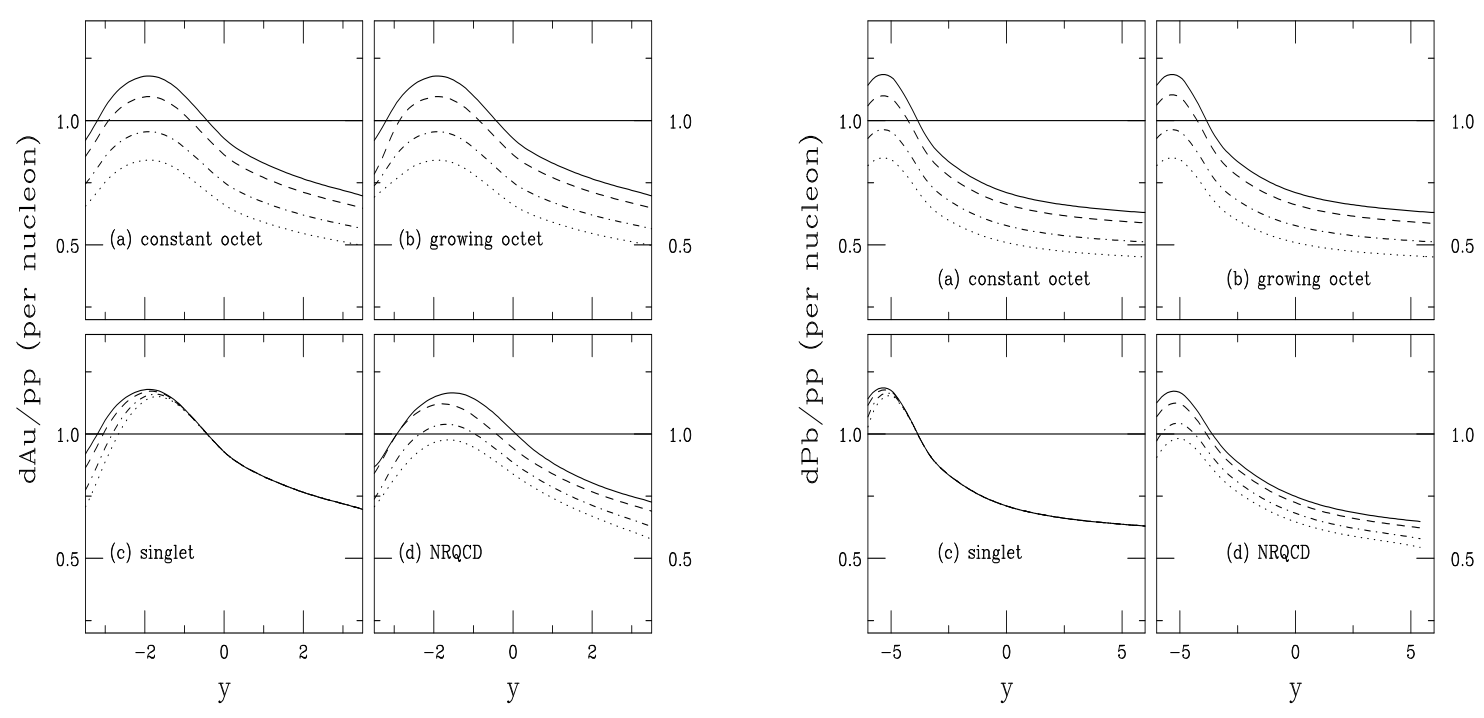

Figure 3. The $J / \psi \mathrm{d} A / p p$ ratio with EKS98 at $200 \mathrm{GeV}$ (left) and $6.2 \mathrm{TeV}$ (right) as a function of rapidity for (a) constant octet, (b) growing octet, (c) singlet, all calculated in the CEM and (d) NRQCD. For (a)-(c), the curves are no absorption (solid), $\sigma_{\text {abs }}=1$ (dashed), 3 (dot-dashed) and $5 \mathrm{mb}$ (dotted). For (d), we show no absorption (solid), $1 \mathrm{mb}$ octet $/ 1 \mathrm{mb}$ singlet (dashed), $3 \mathrm{mb}$ octet $/ 3 \mathrm{mb}$ singlet (dot-dashed), and $5 \mathrm{mb}$ octet $/ 3 \mathrm{mb}$ singlet (dotted).

absorption is included, the trend of the impact parameter dependence is in agreement with the PHENIX data at $y>0$ (the north muon arm) but is too weak to describe the strong dependence at $y<0$ (the south muon arm), see Pereira's talk [2].

\section{Conclusions}

In summary, the RHIC $\mathrm{d}+\mathrm{Au}$ data on open charm and $J / \psi$ are beginning to come into their own. While the QCD calculations agree well with the shape of the STAR $p_{T}$ distributions, they underestimate the reported total cross section. In contrast, the $J / \psi$ cross section is in relatively good agreement with QCD predictions [2] and the agreement of the minimum bias data with calculations including shadowing and nucleon absorption is quite good. Work is ongoing to better understand the impact parameter dependence.

[1] Kollegger $\mathrm{T}$, these proceedings

[2] Pereira $\mathrm{H}$, these proceedings

[3] Nason P, Dawson S and Ellis R K 1988 Nucl. Phys. B 303607

[4] Kidonakis N, Laenen E, Moch S and Vogt R 2003 Phys. Rev. D 67074037

[5] Kidonakis N and Vogt R 2004 Eur. J. Phys. C 36201

[6] Vogt R 2003 Heavy Ion Phys. 1775

[7] McGaughey P L et al. 1995 Int. J. Mod. Phys. A 102999

Vogt R (Hard Probe Collaboration) 2003 Int. J. Mod. Phys. E 12211

[8] Frixione S, Mangano M L, Nason P and Ridolfi G 1998 Adv. Ser. Direct High Energy Phys. 15 609

[9] Adcox K et al. (PHENIX Collaboration) 2002 Phys. Rev. Lett. 88192303

[10] Adams J et al. (STAR Collaboration) 2004 Preprint nucl-ex/0407006

[11] Botner O et al. (UA1 Collaboration) 1990 Phys. Lett. B 236488 
[12] Acosta D et al. (CDF II Collaboration) 2004 Phys. Rev. Lett. 91241804

[13] Huang $\mathrm{H}$, these proceedings

[14] Vogt R, Brodsky S J, and Hoyer P 1992 Nucl. Phys. B 383643

[15] Mangano M L, Nason P and Ridolfi G 1993 Nucl. Phys. B 405507

[16] van Leeuwen $\mathrm{M}$, these proceedings

[17] Cacciari M and Nason P 2003 J. High Energy Phys. 09006

[18] Tai A, private communication 2004

[19] Kharzeev D and Tuchin K 2004 Nucl. Phys. A 735248

[20] Klein S R and Vogt R 2003 Phys. Rev. Lett. 91142301

[21] Thews $\mathrm{R} \mathrm{L}$, these proceedings

[22] Cortese $\mathrm{P}$, these proceedings

[23] Vogt R 2002 Nucl. Phys. A 700539 